\title{
Time-of-flight grazing incidence small angle neutron scattering on Gd nanowires
}

\author{
W. Kreuzpaintnera , J.-F. Moulin, D. Lott, R. Kampmann, M. Haese-Seiller, M. Störmer, \\ and A. Schreyer \\ GKSS Forschungszentrum GmbH, Max-Planck-Straße 1, 21502 Geesthacht, Germany
}

\begin{abstract}
A Gd nanowire grating with a periodicity of approximately $225 \mathrm{~nm}$ prepared on a faceted $\alpha-\mathrm{Al}_{2} \mathrm{O}_{3}$ substrate was investigated by Time-Of-Flight Grazing Incidence Small Angle Neutron Scattering. These measurements allowed to obtain information on the critical wavelength, the reflected and transmitted intensities and also allowed a reconstruction of large portions of the grating truncation rods in reciprocal space. Results were obtained for various orientations around the sample normal and compared with a theoretical model, showing good agreement.
\end{abstract}

\section{Introduction}

With the evolution of nanostructuring processes, Grazing Incidence Small Angle X-ray and Neutron Scattering (GISAXS and GISANS) have established as methods for the analysis of laterally structured surfaces with feature sizes and periodicities of only a few to several hundreds of nanometers [1,2]. In general such experiments are performed with quasi monochromatic beams (typically $\Delta \lambda / \lambda<10 \%$ ) [1,3]. In the case of x-rays, due to high absorption, one is limited to the scattered intensities above the sample horizon, while for neutrons the full pattern, including the transmitted region can be recorded. In GISANS, however, transmitted intensities are nevertheless often not evaluated due to highly complicated transmission functions and absorption effects [3]. In this paper we will show the advantages of faster data acquisition by use of a continuous neutron wavelength spectrum over a single wavelength setup in investigating a periodically laterally structured sample by GISANS and also show that for neutrons the transmitted scattered intensities can be considered for sample analysis.

\section{Experimental results and discusssion}

\subsection{Sample preparation}

A nanostructered surface grating was prepared by Ultra-High-Vacuum (UHV) electron-beam evaporation of $\mathrm{Gd}$ onto a pre-structured faceted $\mathrm{Al}_{2} \mathrm{O}_{3}$ substrate under a shallow angle of incidence. In our particular case the miscut of the $\mathrm{Al}_{2} \mathrm{O}_{3}$ (10) 10$)$ (m-plane) substrate was $0.1^{\circ}$ as determined by x-ray rocking scans. The substrate was annealed in air in a three step process: starting with the furnace and the sample at room temperature the temperature was ramped to $750{ }^{\circ} \mathrm{C}$ at $5^{\circ} \mathrm{C} / \mathrm{min}$, followed by a dwell time of $30 \mathrm{~min}$ after which the temperature was increased to $1500{ }^{\circ} \mathrm{C}$ at a rate of $10^{\circ} \mathrm{C} / \mathrm{min}$. The temperature of $1500^{\circ} \mathrm{C}$ was then held for $24 \mathrm{~h}$ before it was reduced to $750^{\circ} \mathrm{C}$ at $10^{\circ} \mathrm{C} / \mathrm{min}$. After another $30 \mathrm{~min}$ the temperature was ramped to room temperature at $5^{\circ} \mathrm{C} / \mathrm{min}$. This process was found to give a surface pattern with the least defects.

\footnotetext{
a e-mail: Wolfgang.Kreuzpaintner@gkss.de
} 


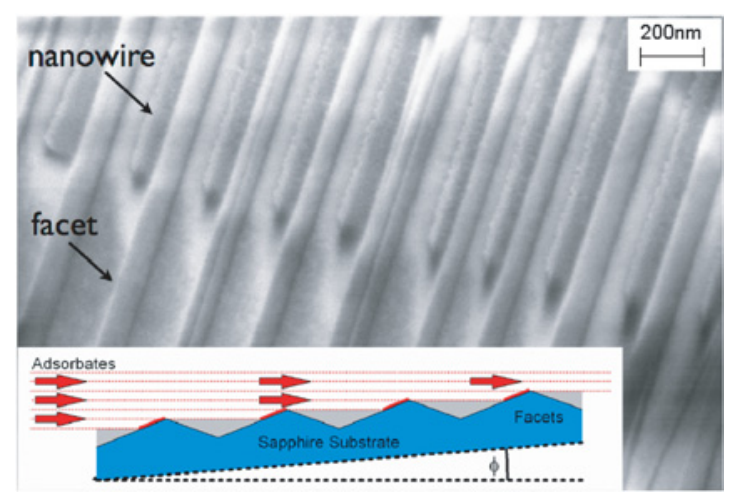

Fig. 1. SEM image of the nanowires, grown by geometrical self shading of a faceted substrate. During the deposition process, a dust particle shaded off a larger area of the substrate such that no deposition occurred in its shade. This sample defect allows to directly see the difference between the substrate without nanowires and the area where nanowires were grown. The inset graph shows the schematic deposition geometry with the periodic selfshading of the substrate by its surface features.

Details on the thermodynamics of the sapphire structuring process and on the sample preparation method are described in [4,5] and references therein. Deposition of $50 \mathrm{~nm}$ Gd occurred at a base pressure of $2 \times 10^{-10}$ mbar and a working pressure of $3 \times 10^{-8}$ mbar at a deposition rate of $1 \AA / s$ under an angle of $5^{\circ}$. The self-shading effect of neighboring facets allows deposition of Gd only on the upper edges of each facet, resulting in the growth of nanowires with a periodicity of approximately $225 \mathrm{~nm}$ and a cross-section of approximately $50 \mathrm{~nm} \times 50 \mathrm{~nm}$. A Scanning Electron Microscopy (SEM) image of the sample surface and the deposition geometry are shown in Fig. 1. In order to prevent oxidization of $\mathrm{Gd}$ a $25 \mathrm{~nm}$ thick Al layer was additionally sputter deposited at a rate of $2.5 \AA / s$ as capping layer. This Al capping layer does not significantly influence the observed intensity distribution and is therefore neglected in the sample analysis.

\subsection{Sample analysis}

GISANS was performed at the combined horizontal REFlectometer and Small Angle Neutron Scattering setup REFSANS at the FRM II neutron source in Garching with a point collimated primary neutron beam of $4 \mathrm{~mm}$ in diameter and a primary integral beam intensity of 400 counts per second. The sample to detector distance was $11710 \mathrm{~mm}$ with the approximately $500 \mathrm{~mm} \times 500 \mathrm{~mm}$ large detector positioned at such a height that the primary neutron beam was just below the lower edge of the detector. The detector resolution is defined by the detector channel size of $2.7 \mathrm{~mm} \times 2.7 \mathrm{~mm}$. The incidence white neutron beam was pulsed by a chopper at a repetition rate of $10 \mathrm{~Hz}$ and the bandwidth was limited to $4.5 \AA$ to $18 \AA$. Typical exposure times for the presented results were in the range of approximately $3 \mathrm{~h}$ with detector count rates in the range of 180 counts per second. The Time-Of-Flight (TOF) detection scheme allows the reconstruction of the different $2 \mathrm{D}$ intensity maps as a function of the wavelength $\lambda$ by software. Fig. 2 shows the scattering geometry and the definition of the relevant goniometer head angles. Measurements were performed for various orientations in $\phi$ around the sample normal at a fixed angle of incidence $\alpha_{i}=0.67^{\circ}$.

\subsection{Measurement results}

\subsubsection{Aligned sample}

The critical wavelength at which the chosen angle of incidence corresponds to the critical angle [6] is $9.2 \AA$ as can be seen in Fig. 3 for a nominal sample orientation of $\phi=0^{\circ}$. The 


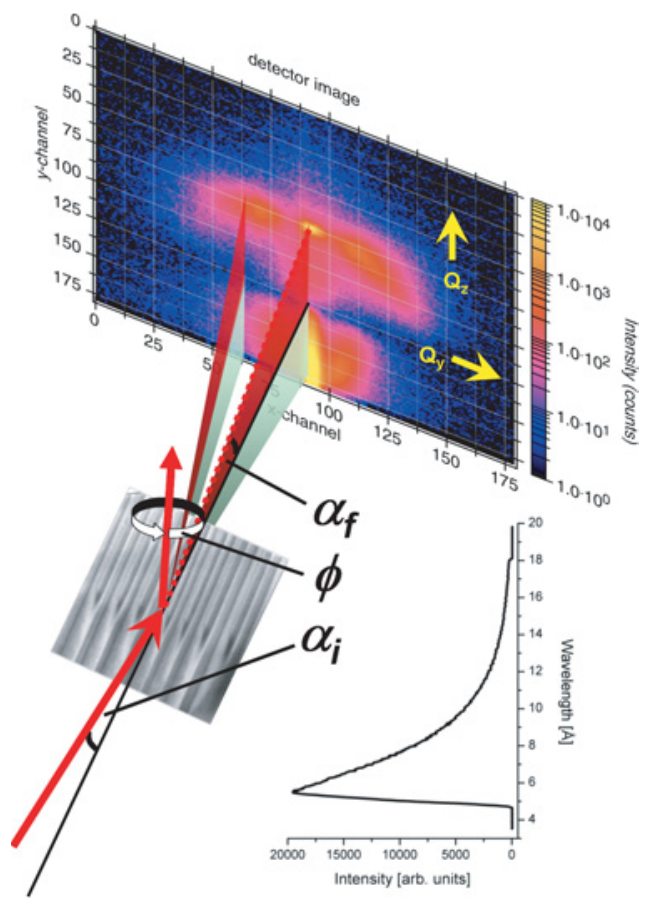

Fig. 2. The applied GISANS scattering geometry: a neutron beam with the shown wavelength distribution in the range from $4.5 \AA$ to $18 \AA$ is shined under a fixed angle of incidence $\alpha_{i}$. The detector image in real space is an integral image over all the wavelengths used and must be investigated in reciprocal space for single wavelength or multiple wavelength evaluation. The sample orientation is defined by $\phi$ around the sample normal with the angles of deflection $\alpha_{f}$ parallel and $2 \theta$ (not sketched) perpendicular to the plane of incidence.

corresponding detector images at wavelengths of $6.9 \AA, 9.2 \AA$ and $10.7 \AA$ are given in Fig. 4 showing intensities at the intersection of the Grating Truncation Rods (GTRs) with the corresponding Ewald sphere. By applying a continuous wavelength spectrum the size of the Ewald sphere is varied in reciprocal space. Hence it is not only possible to reconstruct intersection points but also larger portions of the GTRs by obtaining the integral image over all wavelengths. For this, however, one has to correct each wavelength slice by the background, weight its intensity by the primary beam intensity, correct ballistic effects and add the intensities in reciprocal space. The such reconstructed image of the GTRs is shown in Fig. 5. It is well known, that from the location of the GTRs of a well aligned sample one can directly obtain the periodicity $b=\frac{2 \pi}{\Delta Q_{y}}$ of the lateral structure, which in this case results in a periodicity of approximately $220( \pm 20) \mathrm{nm}$ and hence is in agreement with the periodicity of $225 \mathrm{~nm}$ as obtained from the SEM image.

\subsubsection{Rotated sample}

GISANS on lateral gratings is very sensitive to a rotation of the sample around its normal. The strong influence of the rotation on the scattered detector image is shown in Fig. 6 for a single wavelength slice, showing the scattered intensity distribution at the critical wavelength for transmitted and reflected intensities. The orientations are $\phi=+0.5^{\circ}$ and $\phi=-0.75^{\circ}$ in mathematical direction. The corresponding integral reciprocal space images of the rotated samples are shown in Fig. 7 and Fig. 8 and will be discussed together with the theoretical expectations (2.3.2). 


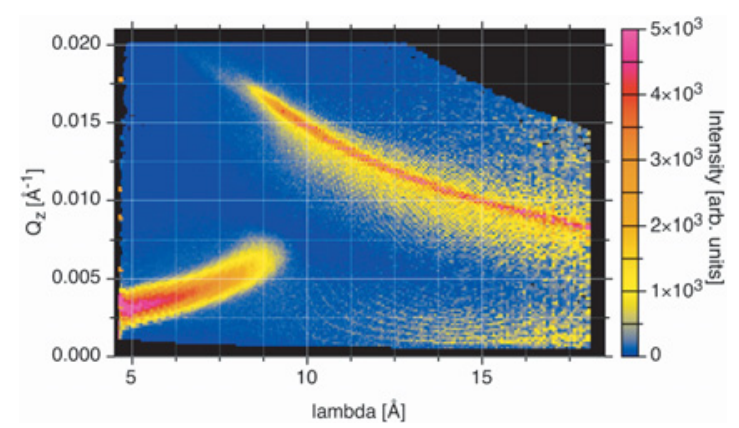

Fig. 3. $Q_{z}$ versus the wavelength $\lambda$ as extracted by integration over $-0.01 \AA^{-1} \leq Q_{y} \leq+0.01 \AA^{-1}$. With increasing wavelength the transition from refracted intensities to reflected intensities occurs with the transition at the critical wavelength of $\lambda \approx 9.2 \AA$.

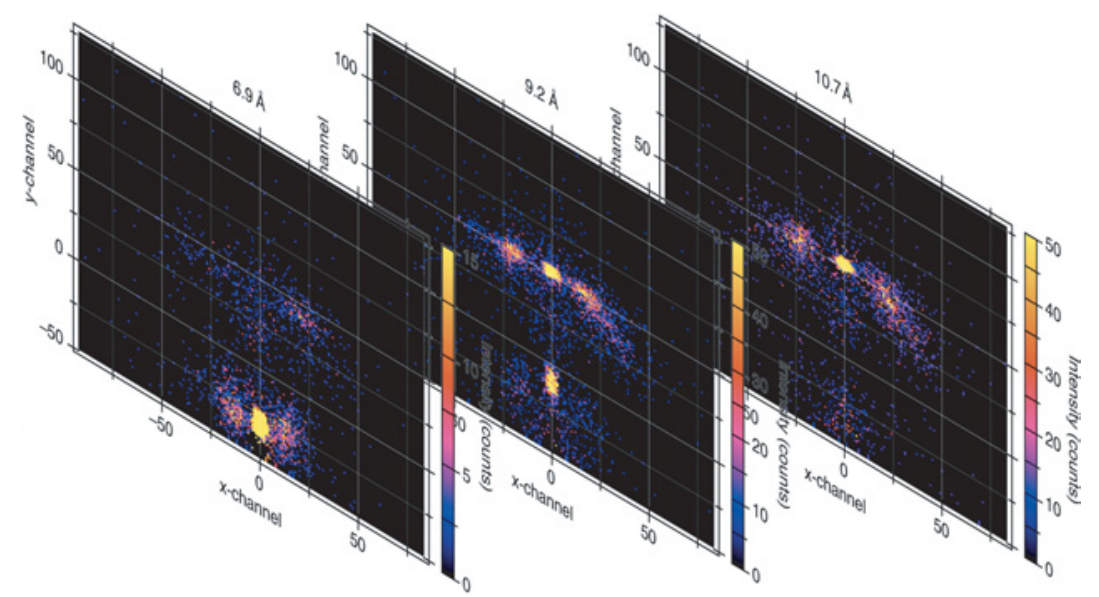

Fig. 4. Detector images for a single wavelength slice which are extractable by the Time-Of-Flight information collected for each neutron. Shown are the detector images for wavelengths of $\lambda=6.9 \AA$ (below the critical wavelength), $9.2 \AA$ (at the critical wavelength) and $10.7 \AA$ (above the critical wavelength).

\subsection{Discussion}

Yan and Gibaud [7] recently published theoretical and experimental results on the intersection of GTRs in reciprocal space with the Ewald sphere using GISAXS. Following their theoretical approach and by also taking the neglected solutions for transmission into account, intensities in reciprocal space are to be expected at

$$
\begin{aligned}
Q_{y}= & \frac{2 \pi n \cos (\phi)}{b}-\frac{2 \pi \sin ^{2}\left(\alpha_{i}\right) \cos (\phi) \sin (\mp \phi)}{\lambda} \\
& \times\left(1 \pm \sqrt{1-\frac{\lambda^{2} n^{2}}{b^{2} \sin ^{2}\left(\alpha_{i}\right) \cos ^{2}(\phi)}\left(1-\frac{2 b \sin (\mp \phi)}{n \lambda}\right)}\right) \\
Q_{z}= & \frac{2 \pi \sin \left(\alpha_{i}\right) \cos \left(\alpha_{i}\right) \cos (\phi)}{\lambda}\left(1 \pm \sqrt{1-\frac{\lambda^{2} n^{2}}{b^{2} \sin ^{2}\left(\alpha_{i}\right) \cos ^{2}(\phi)}\left(1-\frac{2 b \sin (\mp \phi)}{n \lambda}\right)}\right) .
\end{aligned}
$$

where $\lambda$ denotes the neutron (or x-ray) wavelength, $\alpha_{i}$ the angle of incidence, $\phi$ the angle of the sample orientation around its normal with $\phi=0$ being the case in which the nanowires on the sample are aligned parallel to the plane of incidence of the primary beam, $b$ is the period of the nanowire grating and $n$ is the order of the scattered intensities. In both formulas, the positive 


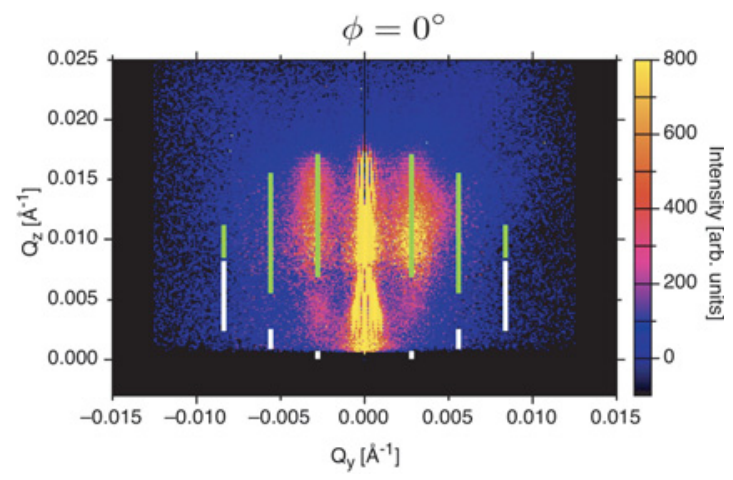

Fig. 5. The reconstructed integral reciprocal space image for a nominal sample orientation of $\phi=0^{\circ}$ with the intensities obtained for all applied wavelengths plotted together with theoretically expected locations of the intensities for reflected (green) and transmitted (white) neutrons. A large portion of the grating truncation rods can be reconstructed allowing for a fast assessment of the lateral structure.
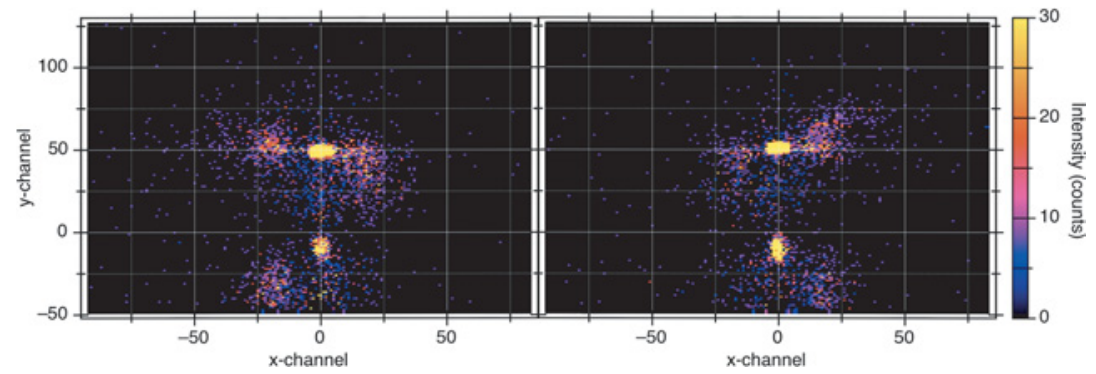

Fig. 6. The detector images at the critical wavelength of $\lambda=9.2 \AA$ obtained for orientations around the sample normal of $\phi=+0.50^{\circ}$ (left) and $\phi=-0.75^{\circ}$ (right) showing the drastic influence of only a small sample rotation onto the location of the intersection of the GTR with the Ewald sphere.

sign before the root accounts for reflected intensities, whilst the negative sign accounts for the transmitted intensities with the opposite relationship for the sign in $\sin (\mp \phi)$. As neutrons also penetrate the sample after scattering on the nanowires both solutions should be considered. As will be seen, however, these formulas only describe parts of the scattered intensity distribution. Especially the transmitted intensities in the vicinity of the sample horizon lie outside the value range of these formulas.

\subsubsection{Aligned sample}

In order to compare the positions of the experimentally collected portions of the GTRs to theory, calculations were performed using above formulas (1) and (2). The applied wavelength spectrum above and below the critical wavelength, as obtained from the plot in Fig. 3 was taken into account and a real space grating periodicity of $225 \mathrm{~nm}$ was assumed for the calculations. The such calculated intensity positions up to $3^{\text {rd }}$ order GTRs for reflected and transmitted neutrons are plotted into the corresponding Fig. 5 for direct comparison with experimental data. The length and the positions of the GTRs can be well described for reflected neutrons. However, the calculated positions of transmitted intensities are not obtainable with the experimentally applied measurement parameters as they would lie below the lower edge of the detector for this sample orientation. It is apparent that the above formulas do not describe the transmission areas in reciprocal space in the vicinity of the sample horizon where intensities that extend the GTRs of reflected neutrons into the regime below the sample horizon are visible. For explanation of this intensity distribution refraction corrections and more sophisticated theoretical methods must be used. Close to the sample horizon, Yoneda peaks [8] are expected (and visible) and 


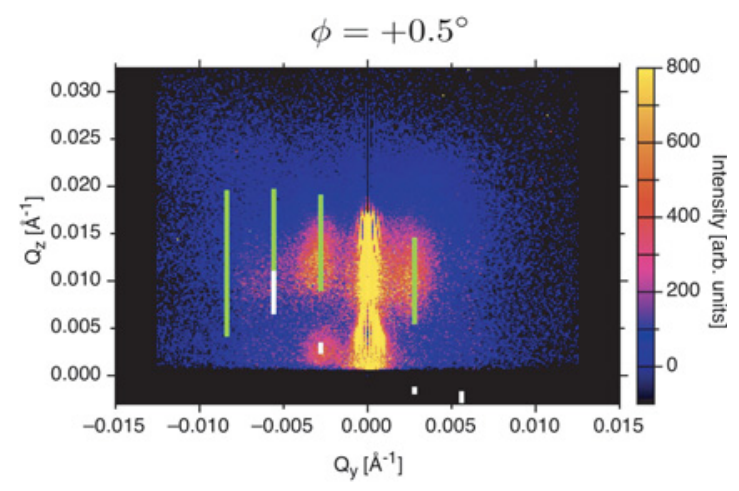

Fig. 7. The reconstructed integral reciprocal space image for a nominal sample orientation of $\phi=$ $+0.50^{\circ}$ plotted together with theoretically expected locations of the intensities for only reflected (green) and also transmitted (white) neutrons.

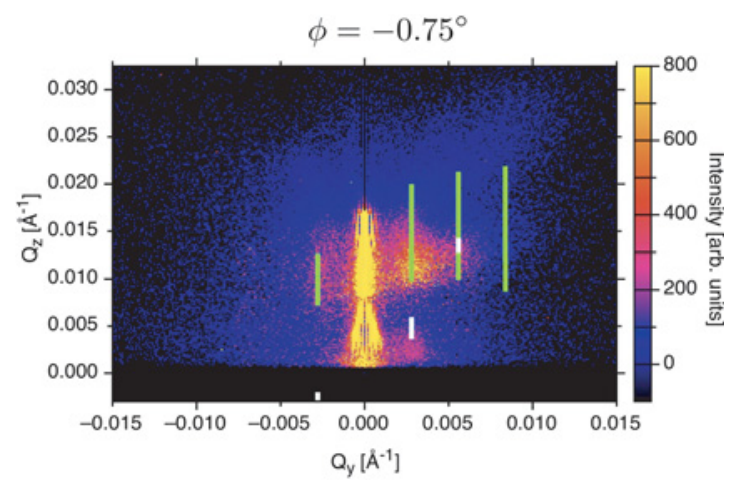

Fig. 8. The reconstructed integral reciprocal space image for a nominal sample rotation state of $\phi=-0.75^{\circ}$ plotted together with theoretically expected locations of the intensities for reflected (green) and transmitted (white) neutrons.

the transmission function is also expected to be complicated for the transmitted intensities, therefore a Distorted Wave Born Approximation (DWBA) approach as used in $[9,10]$ would be necessary to describe the observations but is beyond the scope of this paper.

\subsubsection{Rotated sample}

Due to the term $\frac{2 b \sin (\mp \phi)}{n \lambda}$ in the root of equations (1) and (2) the GISANS pattern is very sensitive to a rotation of the grating around the plane of incidence. As a consequence, the accessible portions of the GTRs also change very asymmetrically around the scattering plane for a given wavelength distribution. The theoretically expected GTRs that were calculated analogous to the case of the non-rotated sample are plotted together with the experimental results in Fig. 7 and Fig. 8. In these two investigated cases, the theoretical fits are less precise for the intensity distributions above the sample horizon, but in this scattering geometry the first order of transmitted intensities is accessible and their location in reciprocal space is in good agreement with the theoretical predictions.

\section{Conclusion}

We have shown that TOF is a very powerful technique for extending the use of Grazing Incidence Small Angle Neutron Scattering as it allows for the use of a basically white neutron beam. This 
makes it possible to collect in a single measurement a more complete reciprocal space image than can be obtained by a single wavelength measurement. By our measurement method we are further able to directly obtain the critical wavelength and can also analyze single wavelength GISANS results. Our first results were evaluated and found to be in good agreement with theoretical expectations. It should be noted that a full quantitative analysis would allow to obtain not only the position of the GTRs but information on all sample surface characteristics, including lateral structure sizes, shapes and periodicities and variations thereof as a statistical average from the entire illuminated sample surface convoluted with the instrumental resolution. Additional GISANS investigations with a pulsed and nearly white neutron beam, including the magnetic properties of the investigated Gd nanowire sample will be presented elsewhere.

\section{References}

1. M. Pannetier, F. Ott, C. Fermon, Y. Samson, Physica B 335, 54 (2004)

2. G.T. Baumbach, D. Lübbert, U. Pietsch, N. Darowski, L. Leprince, A. Talneau, J. Schneck, Physica B 248, 343 (1998)

3. P. Müller-Buschbaum, J.S. Gutmann, R. Cubitt, W. Petry, Physica B 350, 207 (2004)

4. M. Huth, K.A. Ritley, J. Oster, H. Dosch, H. Adrian, Adv. Funct. Mater. 12, 333 (2002)

5. A. Westphalen, H. Zabel, K. Theis-Bröhl, Thin Solid Films 449, 207 (2004)

6. A.-J. Dianoux, G. Lander (eds.), Neutron Data Booklet, 2nd edn. (Institut Laue-Langevin, France, 2003), pp. 3.2-8

7. M. Yan, A. Gibaud, J. Appl. Cryst. 40, 1050 (2007)

8. Y. Yoneda, Phys. Rev. 131, 2010 (1963)

9. P. Mikulik, T. Baumbach, Physica B 248, 381 (1998)

10. P. Busch, D. Posselt, D.-M. Smilgies, M. Rauscher, C.M. Papadakis, Macromolecules 40, 630 (2007) 